\title{
The human eye: a model system for teaching optics
}

\section{Vasudevan Lakshminarayanan}

Vasudevan Lakshminarayanan, "The human eye: a model system for teaching optics," Proc. SPIE 9666, 11th Education and Training in Optics and

Photonics Conference, 96661D (5 June 2009); doi: 10.1117/12.2207966

SDIE Event: Eleventh International Topical Meeting on Education and Training in Optics and Photonics, 2009, St. Asaph, United Kingdom 


\title{
The Human Eye: A Model System For Teaching Optics
}

\author{
Vasudevan Lakshminarayanan \\ University of Waterloo \\ Depts. Of Physics and Electrical Engineering \\ and School of Optometry \\ Waterloo, Ontario, Canada N2L 3G1 \\ Email: vengu@uwaterloo.ca
}

\begin{abstract}
The human eye treated as an optical system is an excellent model to teach various aspects of Optics. The range of topics treated can start from simple refraction upto quantum optics including detection of single photons. In particular, concepts and methods such as ray tracing, apertures and stops, field of view calculations, gradient index systems, non-centered systems, Fraunhofer and Fresnel diffraction, scattering, Fourier optics as well as aberrations (both Seidel and wavefront aberrations) and adaptive optics can be illustrated using various properties of the human visual system. A course such as this will be of interest to students from biological sciences as well natural science and engineering students who desire some "real world" applications. I have taught these aspects and will describe my experiences and a model curriculum utilizing this ansatz.
\end{abstract}

Keyword list: Optics, dioptrics of eye, biomedical optics, inter-disciplinary courses

\section{INTRODUCTION}

It is generally said that the eyes are the windows to the soul. I paraphrase this saying by stating that the human eye is the window to the world of optics. The eye offers a wonderful system for teaching at various levels of sophistication different aspects of optics. Traditional textbooks, i.e., introductory physics textbooks, such as those by Serway and Beichner ${ }^{1}$, Halliday, Resnick and Walker $^{2}$ or Giancoli ${ }^{3}$ often treat the eye as a model application and deal with concepts such as myopia (short-sightedness) and hyperopia (long-sightedness). Advanced courses devoted exclusively to optics, do not spend much time (if any at all) to the eye as an optical instrument. Even the classic book by Jenkins and White ${ }^{4}$ devotes about three pages in its chapter on optical instruments. The one exception is the book by Keating ${ }^{5}$. In this book, the human eye is used throughout in both examples and problems. However, Keating's book is rather specialized, and is meant to be used in optics courses taught in Optometry schools and colleges in the professional program.

The vertebrate, human eye is an excellent example of a decentered optical system which utilizes not only aspherical components, but also gradient index sub-systems. Because, it utilizes biological material, we can also expect it to exhibit significant chromatic aberration. The eye also has an iris which acts as an aperture stop. Image formation by the human eye can be treated as either as a thin lens system or as a thick lens system. The eye suffers from considerable high order aberrations, including (and not limited to) spherical, coma, meridional astigmatism, etc. Finally, the photoreceptors in the retina, the rods and cones, act as classic fiber optic elements, which exhibit waveguiding phenomena. As an optical system, we can define a point spread function and an optical transfer function for the eye and study the imaging characteristics as well as phenomena such as aliasing. The reader is referred to, for example, Atchison and Smith ${ }^{6}$, for a detailed description of the optics of the eye. 
It is possible, to devise a curriculum, based on the eye, that will teach various aspects of optics. A course based on this fundamental assumption, will be of interest to a wide variety of students, ranging from those in the biological sciences, biomedical engineering, biophysics and medical physics, as well as those in allied health sciences such as pre-optometry, ophthalmic nursing, etc.

\section{THE EYE AND SOME EXAMPLES}

\section{Geometric Optics}

Typical optics texts begin with a discussion of Fermat's principle and the concept of wavefronts from which the concept of rays and ray bundles are generated. Following the introduction of the refractive index, we are led to the idea of refraction at an interface between two media. The cornea - air interface can be considered as the model for this; from this, it is possible to deal with total internal reflection. The eye example to use herein is the idea of the viewing of the "angle" which is used in a technique called gonioscopy. One can also use the fact that the photoreceptor captures the incident light and channels them to the site of absorption using the idea of total internal reflection. The crystalline lens is a gradient index medium, and it is possible to teach ray tracing through such media. The iris of the eye, which acts as an aperture, is a good example of both the entrance and exit pupils (image when viewed through the cornea and image when viewed from the retina, after being refracted by the crystalline lens). Curved surface refraction can be thought of as refraction at the various elements (surfaces of the cornea, surfaces of the crystalline lens). Additionally, instead of treating the eye as a thin lens system, we can treat it as a thick lens system and introduce Gaussian points, etc. Another reason to use the eye as a model system is the fact that we can introduce concepts of non-spherical systems (i.e., aspherics) and deal with astigmatic lenses - this concept is rarely taught in traditional optics courses. Because the eye is a decentered system, we can discuss concepts such as prismatic deviation introduced due to a light ray incident at some distance from the optical center (e.g., Prentice's law). Spherical mirrors can be very easily illustrated by the example of Purkinje Sanson images (light reflections seen due to reflections at various structures of the eye, namely the anterior corneal surface, posterior corneal surface, anterior lens surface and posterior lens surface). Here, the ideas of Fresnel losses can be introduced. In a more advanced course, it is possible to construct computational eye models and illustrate imaging characteristics by changing the parameters of the model. It is easy (and much welcomed by students) to use the ABCD matrix formalism ${ }^{7}$ in these exercises.

\section{Physical Optics}

Physical Optics ideas can be taught by using a number of different examples from the visual system. Interference and the Young double slit experiment has an immediate application in the eye - namely to measure what is known as the grating acuity ${ }^{8}$. This is done by projecting two small coherent spots in the entrance pupil of the eye. These spots will now act as the double slit and produce sine-wave gratings on the retina. As in the traditional Young double slit, by varying the separation between the two spots, we can change the spatial frequency of the fringes and find the limit of vision. This can also lead to finding the contrast threshold for different spatial frequencies. As in the traditional interference experiment, here too, we can define the Michelson contrast, etc. This is just one example. From this, we can generalize and define the modulation transfer function (MTF) and the Optical Transfer function (OTF) and hence, study Fourier optics. Diffraction can be taught by studying imaging by different apertures - more specifically by circular apertures and showing how visual acuity varies depending upon the size of the aperture. When we talk about visual acuity, we are dealing with resolution ability and hence the Rayleigh criterion. Of course, other criteria could be used.

Polarization phenomena can be illustrated by using for example, demonstrations of Haidinger's brushes. This entoptic phenomenon is due to the polarization properties of short wavelength sensitive photoreceptors in the retina ${ }^{9}$. Light scattering can be illustrated by the presence of 
floaters in the eye media and the presence of glare. As noted earlier, photoreceptors in the retina behave as classic fiberoptic properties and demonstrate waveguide modes. ${ }^{10}$

Wavefront aberrations can be measured using, for example, Hartmann Shack sensors, and this is a major area of research in vision science ${ }^{11}$. We can describe adaptive optics techniques and descriptions of wavefront aberrations using Zernike polynomials (as well as comparison with Seidel aberrations) using the aberration measurements of the human eye.

\section{Quantum Optics}

Photon statistics can be illustrated by analyzing the fact that a dark adapted human eye, under some conditions, needs only about 4-7 photons to give a response of "I see a spot of light" about $60 \%$ of the time. In fact, one only needs the absorption of one photon by one photoreceptor to generate a neural response ${ }^{12}$. There is also the presence of dark noise. More recently, it has been proposed that human vision can be used to observe entanglement ${ }^{13}$.

\section{DISCUSSION}

In the above description, I have not dealt with various instrumentation and equipment used to study the visual system. These offer further examples to teach physics and physics concepts. Additional methods that can be taught include topics such as feedback control, optical engineering, and so forth. Various aspects of imaging science can also be introduced (optical coherence tomography, functional magnetic resonance imaging, etc.). An example of a course that uses the eye in a significant manner to teach physics/optics in particular and science in general is described by Falk, Brill and Stork. ${ }^{14}$ This course, at the University of Maryland, College Park, was taught to non-science majors.

I have taught physical optics and geometric optics courses using the eye as a model system. A caveat is that these courses were taught to professional Optometry students in their first , postundergraduate, professional year. These courses form the bedrock for their future career as optometrists. However, other students, such as those majoring in physics have taken these courses. These courses, taught at both the University of Waterloo and at the University of Missouri - St. Louis (where I was previously affiliated) were also accompanied by a laboratory. Many of the laboratory exercises used aspects of human vision. Because of the application oriented approach, there is considerable interest and my student evaluations have always praised the practical applications shown. I believe a course such as this, would be of great interest to various categories of students and not just limited to future optometry professionals. 


\section{REFERENCES}

[1] R.A.Serway, R.J.Beichner, Physics for Scientists and Engineers with Modern Physics, $5^{\text {th }}$ Edition, Volume 2, Saunders, Ft. Worth, 2000.

[2] D. Halliday, R.Resnick and J. Walker, Fundamentals of Physics, $8^{\text {th }}$ edition, Wiley, New York, 2007.

[3] D.C.Giancoli, Physics: Principles with Applications, $6^{\text {th }}$ edition, Prentice Hall, New York, 2004. [4] F.A.Jenkins and H.E.White, Fundamentals of Optics, $4^{\text {th }}$ edition, Chapter 10, McGraw Hill, New York, 1976.

[5] M.P.Keating, Geometric, Physical and Visual Optics, $2^{\text {nd }}$ edition, Butterworth Heinemann, Boston, 2002.

[6]D.A.Atchison and G.Smith, Optics of the Human Eye, Butterworth Heinemann, oxford, UK, 2000.

[7] see for example, G.Kloos, Matrix Methods for Optical Layout, SPIE Press, Bellingham, WA, 2007.

[8] see for example, C. Roychoudhuri, V. Lakshminarayanan, Role of the retinal detector array in perceiving the superposition effects of light, in The Nature of Light: Light in Nature, ed. K. Creath, Proc. SPIE, 6285, 628507, 2006.

[9] A. LeFloch, G.Ropars, J.M.Enoch, V. Lakshminarayanan, Polarization sense in human vision, Science, submitted, 2009

[10] V. Lakshminarayanan and J.M. Enoch, Biological Waveguides, Handbook of Optics, Volume 3, Chapter 10, McGraw Hill, NY, 2000.

[11] G.Dai, Wavefront Optics for Vision Correction, SPIE Press, Bellingham, WA., 2008

[12] see for example, . V. Lakshminarayanan, Vision and the single photon, in, "What is a

Photon?" ed. C.Roychoudhuri and K. Creath, Proc. SPIE, 5866:332-337, 2005.

[13] P.Sekatski, N.Brunner, C.Branciard, et al., Quantum experiments with human eyes as detectors based on cloning via stimulated emission, arXiv:0902.2896, 2009.

[14] D.Falk, D.Brill and D.Stork, Seeing the Light, Wiley, NY, 1986. 